\title{
Numerical Analysis of Polymer Composites for Actuation
}

\author{
Shivashankar Hiremath ${ }^{1 *}$, Vidyashree Sangappa ${ }^{2}$, Sangamesh Rajole $^{3}$, Satyabodh Kulkarni ${ }^{4}$ \\ ${ }^{1}$ Department of Mechatronic Engineering, Manipal Institute of Technology, Manipal Academy of Higher Education, Manipal, \\ Udupi 576104, India \\ ${ }^{2}$ Department of Computer Science and Engineering, Bangalore Institute of Technology, Bangalore 560004, India \\ ${ }^{3}$ Department of Mechanical Engineering, Central University of Karnataka, Kalaburgi 585367, India \\ ${ }^{4}$ Department of Mechanical Engineering, National Institute of Technology Surathkal, Mangalore 575025, India
}

Corresponding Author Email: ss.hiremath@manipal.edu

https://doi.org/10.18280/rcma.305-603

Received: 20 December 2019

Accepted: 14 October 2020

\section{Keywords:}

thermal actuator, composite beam, temperature, polymer material, numerical analysis

\begin{abstract}
The design of a polymer composite actuator is essential for micro and nano applications. Thus, the composite material may deform or deflects as specific stimuli are applied, such as heat, electrical, light source, etc. The deformation of the composite material is caused by the type of stimulus applied. Hence, while it is heated, the expansion takes place quickly, and the heating is shut down, the material shrinks very slowly. In the present investigation, this phenomenon is mainly studied in the actuation of composite beams. Numerical analysis of carbon black filled polymer composite beam expansion, and contraction is being analyzed in this research. The structure of the beam has been created, and the composite properties are incorporated into the beam, and the uniform heat source is applied on to the surface of the beam. The heating and cooling of the composite material predict the increase and decrease in the temperature of the beam. The numerical analysis of the temperature-dependent expansion and contraction of the composite beam has been carried out successfully. An increase in temperature is observed to signify the slight expansion in the composite beam, whereas the contraction of the composite beam takes a longer time to reach room temperature. Also, the increase in the content of the filler leads to a decrease in the expansion of the composite beam. The numerical simulation of the polymer composite thus provides a solid platform for the experimental study of thermal actuators.
\end{abstract}

\section{INTRODUCTION}

The material can convert different types of stimuli, such as thermal energy, light energy, electric energies into mechanical deflection, motion, and expansion/contraction. In that thermal energy, one derived from the heat source, and it takes a shorter time to heat the material and a longer time to cool the material, because of the natural convection. The different energy sources can be easily converted into thermal energy. Such as light source is abundant and freely available in nature. The light energy can easily turn into the thermal form. Like that, electric power also quickly transforms into the heat form based on the types of electrical sources. Thus, thermal energy is one, which is a valuable source and easily formable/available for any actuation or movement of the objects. Thermal energy is not available directly; it needs material or medium to form heat. In that material is one of the elements which can convert light energy to thermal and electric power to thermal. The photothermal and electrothermal conversion finally leads to sensing and actuation applications [1-4].

Growth in the use of different materials such as metal, ceramic, polymer, and composite has reached a level where they are now challenging the use of conventional material. The use of these materials in a wide variety of applications is due to their many outstanding physicals, thermal, chemical, and mechanical properties. Thus, the material with the varied composition is significant in the development of a device like a microswitch, soft robots, micro-relays, and energy harvesting [5-8]. The material with excellent thermal absorption and quick releasing thermal energy is challenging in the conventional material. Metal material is the one good conductor, which converts light or electric energy into the thermal. Also, metals are transfer heat immediately and cool as quickly as possible due to natural convection. The ceramic materials are holding the thermal energy for a longer time as well as ceramics material conducts some extent. The polymer materials are the non-conducting material, needs more energy to convert into the heat. Finally, the composite materials are one which can overcome the drawbacks of the conventional material by tailoring the appropriate properties of the materials. In that polymer composite, materials can be easily prepared, modified, less cost, and ease of availability for the experimental point of view. Also, polymer composite materials have equal importance to the design and development of the actuators.

Nowadays, researchers have been extensively involved in the development of polymer-based applications $[9,10]$. The use of polymer has grown at a phenomenal rate for the last four decades. This material has impressive and diverse range applications in aircraft, boats, automobiles, civil constructions, 
sports components, and consumer goods. The polymer material can not be used alone, so some of the filler material was needed, and it becomes the composite. Composites are a promising material for the development of a new type of material as well as applications. The polymer composite is embedded with different conductive filler materials such as carbon black, carbon nanotube [11, 12], graphite, graphene oxide and graphene [13], which appear in the recent interest of thermal actuation. The need for the actuation is the energy source, geometry, and material. The energy source has an important phenomenon in the actuation. The actuator can be controlled by increasing and decreasing the energy sources. The geometry is another parameter that influences the actuation by changing the size of the actuator, such as length, width, and thickness. Finally, the material has a vital role in the development of the actuators. The geometry is built with the help of material properties and controlled the actuation by input source. Thus, the actuation is observed by creating the composite beam or slab and then stimulated by thermal input. Hence, the heating and cooling of composite material can lead to the expansion and contraction of the composite beam [14, $15]$.

In the present study, the numerical analysis of the thermal expansion and contraction of polymer composites is examined using approximate equations. The expansion and contraction equation can be derived from the geometry of the beam and conduction and convection of the composite material. The study involves polydimethylsiloxane as matrix material, which is filled with a carbon black filler material. The beam of the composite was formed by adding a matrix into the carbon black filler. Composite beam heating instantly expands, while shrinking / contraction happens very gradually. Also, the temperature rise and fall were predicted by the analytical method. The heat and cooling phase of the composite beam is calculated and simulated using commercially available software. The actuation beam is analyzed using polydimethylsiloxane and carbon black composite material by assigning material properties. A different volume fraction of $5 \%, 10 \%$, and $15 \%$ of filler material was considered in the present analysis. The volume fraction of material with varied carbon black content is obtained from the rule of mixture.

The numerical study can predict the performance of the composite beam with respect to the expansion and contraction. The study also offers a reference base for the experimental study of thermal actuation based on polymers composite. In continuous with this, numerical study suggests the validating the experimentation on the thermal actuation.

\section{BASIC CONCEPTS}

The primary mechanism for heating of the material is to absorb, reflect, and transmit. The material may absorb the heat and increases its internal or surface temperature. The heat may partially reflect or divert due to the convection phenomenon. Whereas, in the case of transmittance, it may transmit through the material. The numerical analysis, reflectance, and transmittance of the heat on the composite material may be neglected or not considered for analysis, because these effects are very low. Also, the assumption was made such a way that transmittance and reflectance effects on composite material were not included.

The surface of the material absorbs heat energy and raises the temperature of the material. The stimulated thermal input assumed that it passes through the material. The composite beam has a bond structure that links the heat travels between the chain. The increase and decrease in temperature of the material lead to the expansion and contraction in the material $[16,17]$.

The composite structure is created with a dimension of $90 \mathrm{x}$ $14 \mathrm{x} 10 \mathrm{~mm}^{3}$ (length $\mathrm{x}$ width $\mathrm{x}$ thickness). The composite beam contains polydimethylsiloxane polymer and varied volume fraction of carbon black filler material. A constant heat source applied on the surface of the beam is as shown in Figure 1. The heating phase covers the expansion of the beam, and the cooling phase recovers the initial state. The heating and cooling of polymer composite are assumed to be an isothermal object. The composite material properties were calculated using the law of mixture by varying volume percentages of filler content $[18,19]$. These material properties are used in the numerical study of expansion and contraction of the beam.

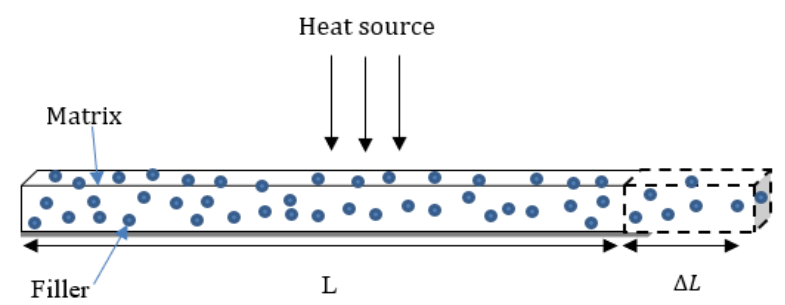

Figure 1. Structure of polymer composite beam

When the heat source is applied on the surface of the polymer composite beam, it leads to an increase in expansion and is relatively very small, is represented as $\Delta \mathrm{L}$. Whereas, in case of heat is off, the expanded beam shrinks by ' $L$ ' as the original length of the composite beam.

\subsection{Material}

The material is an important constituent in the development of polymer based sensors and actuators. In recent past, polymer material like Polyvinylidene difluoride (PVDF) used in energy harvesting [20], polyamide is used in sensor application [21], some of the electronics applications use High-density polyethylene (HDPE) [22], Polyvinyl chloride (PVC) used to detect pollutant [23], Polycarbonate (PC) with Nafion polymer in electrochemical sensor [24], and Poly (dimethylsiloxane) (PDMS) is used for sensing and actuator application [25].

The polymer material is reinforced with ceramic, conductive, and semiconductive particles and made as polymer composite. The different types of filler materials are elaborated in the introduction section. The polymer composites are prepared by solution cast method by varying volume percentage of filler material. The variation of filler can helps to control the specific feature of the polymer composite material. Also, the main polymer material can be easily tailored by adding filler material.

In the present analysis, the main matrix or polymer material is used as polydimethylsiloxane. The polydimethylsiloxane is filled with carbon black material as a filler material. The thermal actuator analysis of polymer composite is carried out by varying the volume percentage of carbon black.

The individual properties of the matrix and filler material are tabulated in Table 1. These properties are used in the analysis of thermal actuators. The varying percentage of 
polymer composites are obtained using the direct rule of mixture. Thus, the material is one of the critical constituents in the proposed beam expansion and contraction of the polymer composite actuator.

\section{NUMERICAL ANALYSIS}

The basic principle of the thermal analysis of the material, heating, and cooling of the polymer composites represented using the equilibrium conduction equation [26, 27]. The transfer of heat flux by conduction is involved in the use of the heat sink as a composite beam and dissipate heat by convection. Heat transfer by conduction means particles of the matter are in direct contact. As heat diffuses through the material, a temperature gradient exists across the material. The equilibrium equation for heat conduction of the material is represented as:

$$
m c_{p} \frac{d T}{d t}=-h A\left(T-T_{a}\right)+\mu q A
$$

where, $m$ is the mass of the beam, and it is calculated using a density of the material and volume of the beam. $c_{p}$, h and $\mu$ are the specific heat capacity, the convective heat transfer coefficient, and the absorption ratio of the material, respectively. Where ' $q$ ' is the constant power/heating source and $T_{a}$ is the ambient temperature or the original temperature before heating. The surface area involved in heating and convection with air is $A$. The heating process of composite beam is expressed mathematically in Eq. (2).

$$
T_{r}=T_{a}+\frac{\mu q}{2 h}\left\{1-e^{\left(-\frac{h A}{m c_{p}}\right) t_{o n}}\right\}
$$

When the surface of the beam is heated with a constant heat source, the temperature of the material's surface rises $\left(T_{r}\right)$ to the maximum at a time $t=t_{\text {on }}$. After some time, heating source shut off will address the decreases in temperature represented by the cooling process in Eq. (3).

$$
T_{f}=T_{a}+\left(T_{m}-T_{a}\right)\left\{e^{\left(-\frac{h A}{m c_{p}}\right) t_{o f f}}\right\}
$$

where, $\mathrm{T}_{\mathrm{f}}$ is fall in temperature of the material from maximum to room temperature during $\mathrm{t}_{\text {off }}$ time. The temperature reached during the heating period is approximately considered as the maximum temperature $\left(\mathrm{T}_{\mathrm{m}}\right)$ of the material.

The expansion and contraction of the composite beam after the heating and cooling process is expressed in Eq. (4) and Eq. (5).

$$
\begin{gathered}
\Delta L_{1}=\alpha\left(T_{r}-T_{a}\right) L \\
\Delta L_{2}=\alpha\left(T_{m}-T_{a}\right) L
\end{gathered}
$$

where, $\alpha$ is the thermal expansion coefficient of the material. The expansion and contraction of the polymer beam is $\Delta L_{1}$ and $\Delta L_{2}$ respectively To ensure that the expansion of the beam can be cooled enough to reach equilibrium temperature, the cooling time should be much higher than the heating time. Also, on carefully observing the above equation, the contraction is approximately $\Delta L_{2}=L$ original length of the beam. This numerical concept is implemented using the MATLAB simulation by varying filler content of the composite beam and observed the expansion/contraction of the actuator.

\section{RESULTS AND DISCUSSIONS}

The thermal actuation depends mainly on the beam's material properties and geometry. Polymer composite with a varied volume fraction filler of $5 \%, 10 \%$, and $15 \%$ is considered in the present study. In the case of the heating phase of the composite material, Figure 2 shows that there is an increase in the volume fraction of the filler leads, an increase in the temperature with time-lapse. It is also observed that $15 \%$ of the filler content of the polymer surface predicts a higher temperature compared to the plane polymer material.

Table 1. The properties of matrix and filler

\begin{tabular}{ccccc}
\hline Material & Density $\left(\mathbf{k g} / \mathbf{m}^{3}\right)$ & Elastic modulus (MPa) & Coefficient of thermal expansion (1/K) & Specific heat capacity $(\mathbf{J} / \mathbf{~ k g . K})$ \\
\hline PDMS & 1030 & 1.84 & $310 \times 10^{-6}$ & 1460 \\
Carbon black & 1890 & 10 & $9 \times 10^{-6}$ & 690 \\
\hline
\end{tabular}

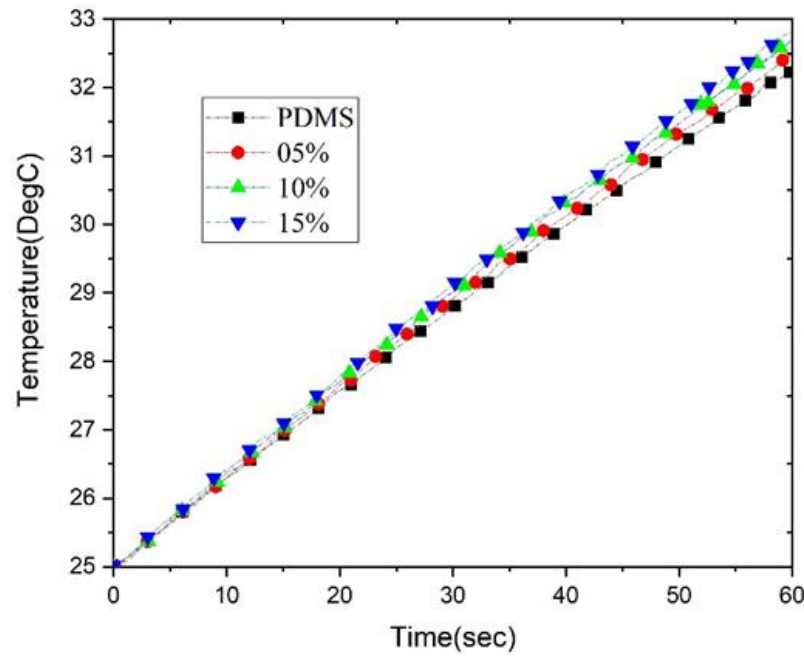

Figure 2. Increase in temperature with respect to time

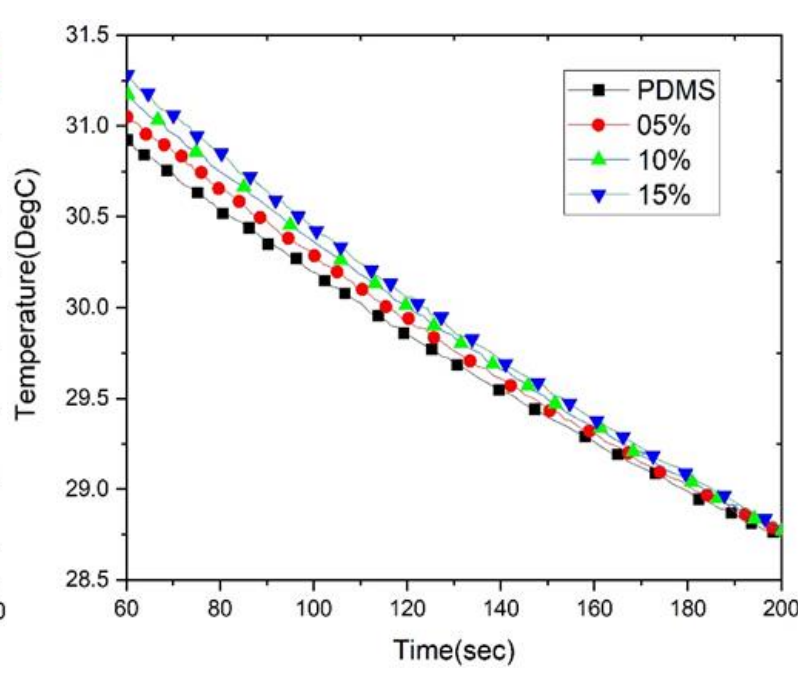

Figure 3. Cooling temperature with respect to time 


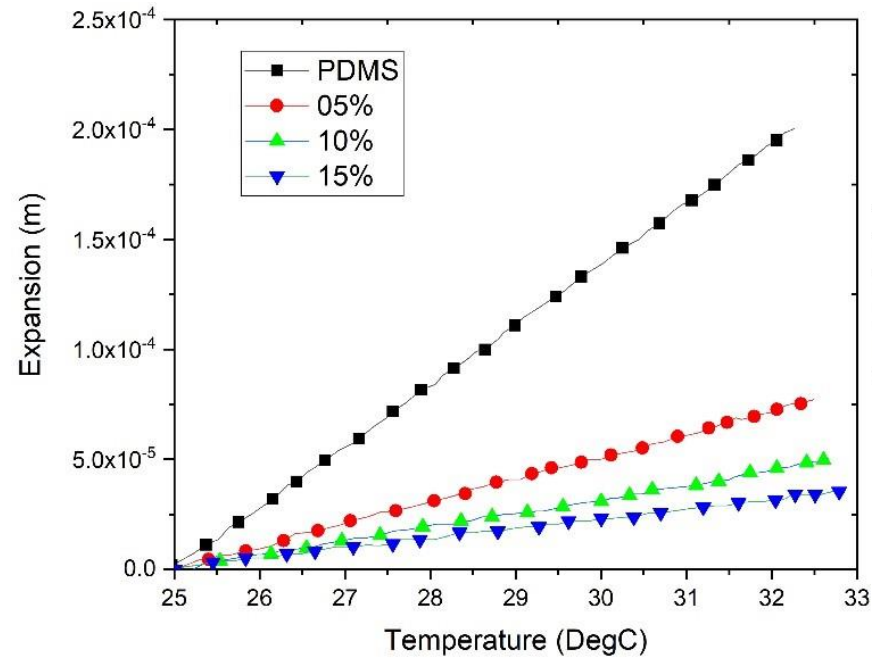

(a)

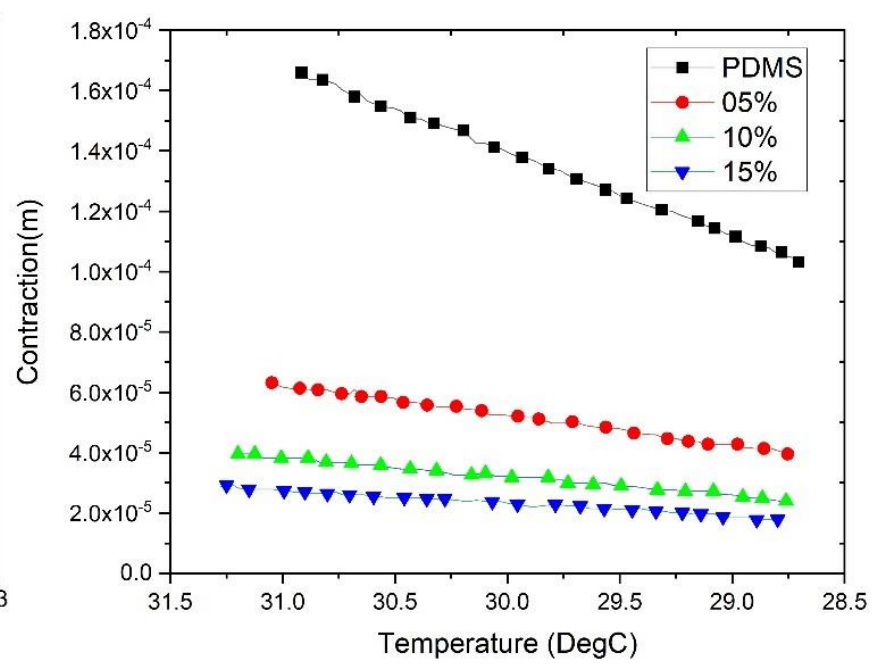

(b)

Figure 4. (a) Expansion of the material with respect to temperature (b) Contraction of the material with respect to temperature

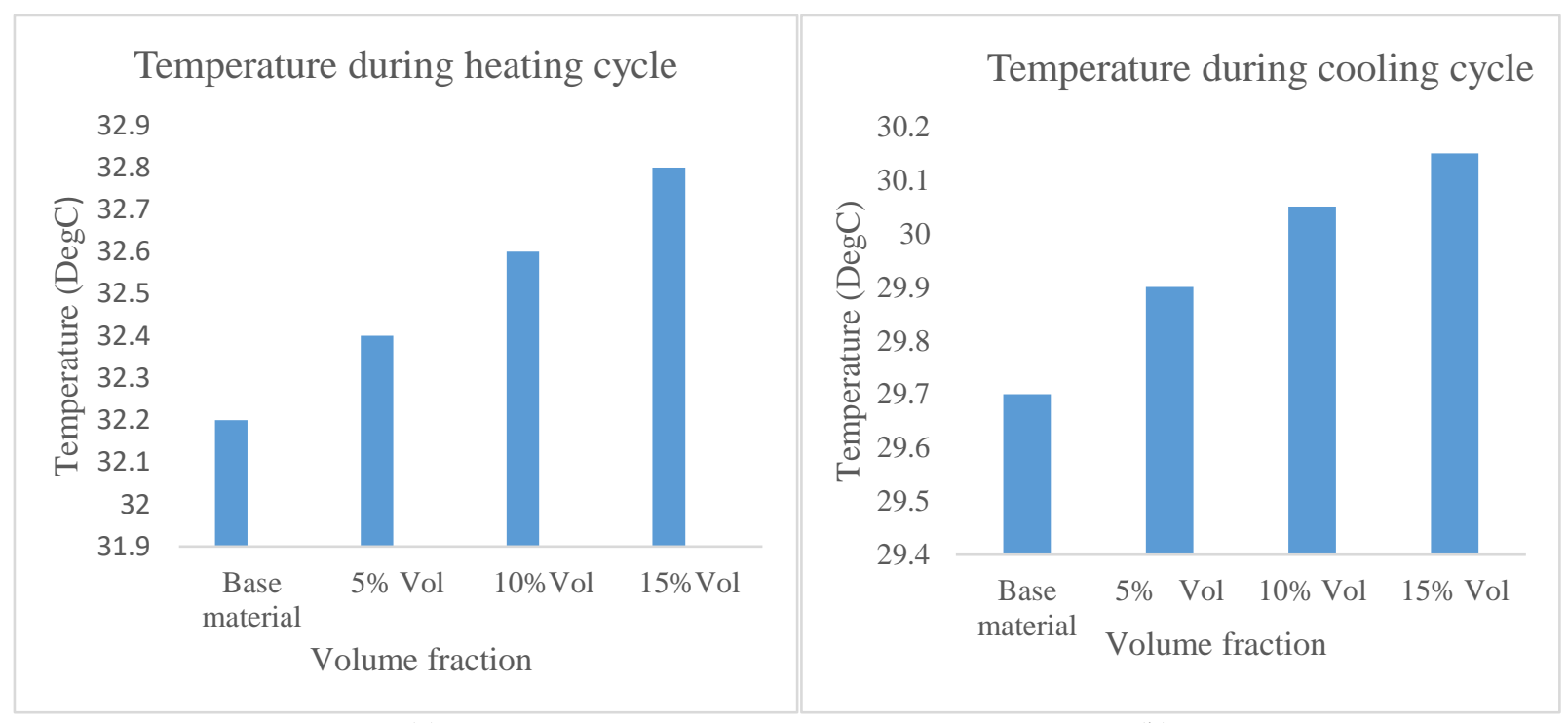

(a)

(b)

Figure 5. Temperature variation with increment of percentage of filler during (a) heating cycle (b) cooling cycle

Whereas in the case of the cooling process, the temperature begins to decrease as the heat is cut off. Figure 3 shows that the time taken for cooling is more than the time taken for heating. It is also observed that the temperature of the pure polymer takes less time than the higher content of the composite filler.

Thermal expansion and contraction of the polymer composites with varying percentages of filler are shown in Figure 4(a) and 4(b).

The expansion of polymer composite material increases with an increase in temperature. Furthermore, it has been found that the higher the volume fraction of carbon black content reduces the expansion. At the same time, shrinkage occurs in polymer composites due to temperature drops from peak to room temperature. It is understood that, due to convection, the cooling phase of the composite material takes place at a prolonged temperature drop.

The composite material with varied volume fraction of carbon black is used to predict the temperature. Heating of the composite material at $60 \mathrm{sec}$, the temperature was noticed. It is observed that, as the filler content of base material is increase with increase in temperature shown in Figure 5(a).
The temperature of the composite material slowly cools during cooling cycle. The base material cools earlier than the varied percentage of carbon content. Figure 5(b) shows the cooling of the composite material predicted at 120th sec, it is understood that, temperature decrease very slowly while cooling phase of the composite material.

The heating of the composite material leads expansion of the Polymer composite. Hence, the base material is the pure polymer, which is expands more compared to varied filler content of carbon black composite. The expansion of the proposed beam decrease as increases in volume fraction is shown in Figure 6(a). During the heating cycle, temperature of the composite increase and expansion of the material decrease.

In the case of cooling cycle, the composite material starts contracts. The base material contracts higher compared to the varied filler content of carbon black. Figure 6(b) shows contraction of the composite during cooling cycle, as a filler varied from $5 \%$ to $15 \%$ leads to contraction from $0.052 \mathrm{~mm}$ to $0.022 \mathrm{~mm}$ at $30^{\circ} \mathrm{C}$. Thus, the expansion and contraction of the polymer composite is mainly depending on the temperature of the beam. 


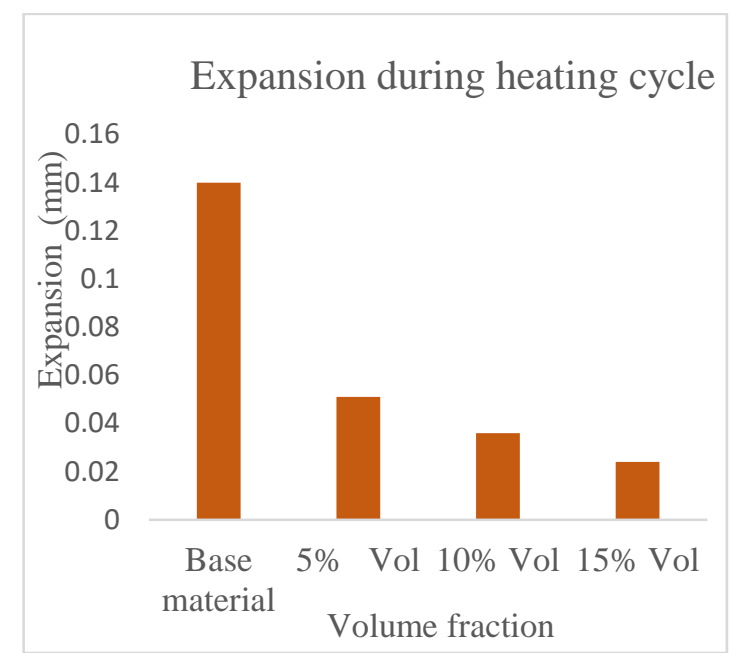

(a)

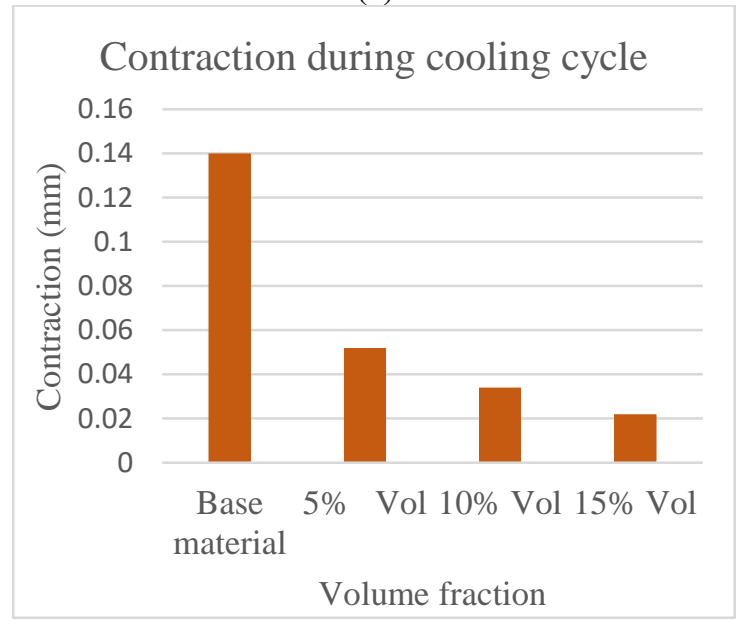

(b)

Figure 6. Displacement of the beam with variation of filler during (a) Expansion (b) Contraction

\section{CONCLUSIONS}

The numerical analysis of temperature-dependent expansion and contraction of the polymer composite were studied. Due to the heating and cooling of the polymer composite beam, minimal expansion and contraction occur. The numerical analysis predicts that the percentage of filler in the pure polymer material will be influenced by increased and decreased in temperature.

Also, It is found that an increase in temperature contributes to a slight expansion of the beam with less time, whereas the shrinkage of the composite material takes a longer time to reach room temperature. The simulation shows sufficient substantial evidence for polymer composite expansion and contraction of the beam. It also required a more mathematical and simulation explanation for the experimental investigation of the thermal actuator. Besides, this analysis can be used in the design and development of applications for micro switches, thermostats, sensors, and actuators.

\section{ACKNOWLEDGMENT}

The authors would like to thank the Department of Mechanical Engineering, National Institute of Technology, Karnataka, for providing a computing facility.

\section{REFERENCES}

[1] Stuart, M.A.C., Huck, W.T.S., Genzer, J., Müller, M., Ober, C., Stamm, M., Minko, S. (2010). Emerging applications of stimuli-responsive polymer materials. Nature $\quad$ Materials, 9(2): 101-113. https://doi.org/10.1038/nmat2614

[2] Zhang, X., Pint, C.L., Lee, M.H., Schubert, B.E., Jamshidi, A., Takei, K., Wu, M. (2011). Optically-and thermally-responsive programmable materials based on carbon nanotube-hydrogel polymer composites. Nano Letters, $11(8)$ : https://doi.org/10.1021/nl201503e

[3] Meng, H., Hu, J. (2010). A brief review of stimulusactive polymers responsive to thermal, light, magnetic, electric, and water/solvent stimuli. Journal of Intelligent Material Systems and Structures, 21(9): 859-885. https://doi.org/10.1177/1045389X10369718

[4] Mishra, S., Unnikrishnan, L., Nayak, S.K., Mohanty, S. (2019). Advances in piezoelectric polymer composites for energy harvesting applications: A systematic review. Macromolecular Materials and Engineering, 304(1): 1800463. https://doi.org/10.1002/anie.201811808

[5] Yeom, S.W., Oh, I.K. (2009). A biomimetic jellyfish robot based on ionic polymer metal composite actuators. Smart Materials and Structures, 18(8): 085002. https://doi.org/10.1088/0964-1726/18/8/085002

[6] Ma, M., Guo, L., Anderson, D.G., Langer, R. (2013). Bio-inspired polymer composite actuator and generator driven by water gradients. Science, 339(6116): 186-189. https://doi.org/10.1126/science.1230262

[7] Xing, H.H., Li, J., Shi, Y., Guo, J.B., Wei, J. (2016). Thermally driven photonic actuator based on silica opal photonic crystal with liquid crystal elastomer. ACS Applied Materials \& Interfaces, 8(14): 9440-9445. https://doi.org/10.1021/acsami.6b01033

[8] Williams, E., Hong, D. (2009). Development of a shape memory alloy composite actuator for the whole skin locomotion robot. In ASME 2009 Conference on Smart Materials, Adaptive Structures and Intelligent Systems, American Society of Mechanical Engineers Digital Collection, pp. 675-683. https://doi.org/10.1115/SMASIS2009-1359

[9] Mirfakhrai, T., Madden, J.D., Baughman, R.H. (2007). Polymer artificial muscles. Materials Today, 10(4): 3038. https://doi.org/10.1016/S1369-7021(07)70048-2

[10] Chronis, N., Lee, L.P. (2002). Polymer-based actuators integrated into microfluidic systems. In Micro Total Analysis Systems 2002, Springer, Dordrecht, 754-756. https://doi.org/10.1007/978-94-010-0504-3_51

[11] Zhou, Z.W., Yan, Q.H., Liu, C.H., Fan, S.S. (2017). An arm-like electrothermal actuator based on superaligned carbon nanotube/polymer composites. New Carbon Materials, 32(5): 411-418. https://doi.org/10.1016/S1872-5805(17)60132-X

[12] Li, C., Thostenson, E.T., Chou, T.W. (2008). Sensors and actuators based on carbon nanotubes and their composites: A review. Composites Science and Technology, 68(6): 1227-1249. https://doi.org/10.1016/j.compscitech.2008.01.006

[13] Huang, Y., Liang, J.J., Chen, Y.S. (2012). The application of graphene based materials for actuators. Journal of Materials Chemistry, 22(9): 3671-3679. https://doi.org/10.1039/C2JM15536B 
[14] Droval, G., Glouannec, P., Feller, J.F., Salagnac, P. (2005). Simulation of electrical and thermal behavior of conductive polymer composites heating elements. Journal of Thermophysics and Heat Transfer, 19(3): 375381. https://doi.org/10.2514/1.12718

[15] Greco, F., Domenici, V., Assaf, T., Romiti, S., Mattoli, V. (2012). Bending actuation of a composite liquid crystal elastomer via direct Joule heating. In 2012 4th IEEE RAS and EMBS International Conference on Biomedical Robotics and Biomechatronics, BioRob 2012, pp.

646-651. https://doi.org/10.1109/BioRob.2012.6290942.

[16] Tant, M.R., McManus, H.L., Rogers, M.E. (1995). Hightemperature properties and applications of polymeric materials. An Overview. https://doi.org/10.1002/(SICI)1097-0126(199701)42

[17] Kim, B., Park, M., Kim, Y.S., Jeong, U. (2011). Thermal expansion and contraction of an elastomer stamp causes position-dependent polymer patterns in capillary force lithography. ACS Applied Materials \& Interfaces, 3(12): 4695-4702. https://doi.org/10.1021/am201118u

[18] Shivashankar, H., Kulkarni, S.M. (2018). Modelling and analysis of thermomechanical behaviour in composite bimorph actuator. In Materials Science Forum, Trans Tech Publications, 928: 209-214. https://doi.org//www.scientific.net/MSF.928.209

[19] Fan, B.H., Liu, Y., He, D.L., Bai, J.B. (2018). Achieving polydimethylsiloxane/carbon nanotube (PDMS/CNT) composites with extremely low dielectric loss and adjustable dielectric constant by sandwich structure. Applied Physics Letters, 112(5): 052902. https://doi.org/10.1063/1.5016543

[20] Yoon, S.J., Arakawa, K., Uchino, M. (2015). Development of an energy harvesting damper using PVDF film. International Journal of Energy Research, 39(11): 1545-1553. https://doi.org/10.1002/er.3357

[21] Sariişik, M., Koncar, V., Cochrane, C., Lewandowski, M., Boussu, F., Dufour, C. (2009). Electro-conductive sensors and heating elements based on conductive polymer composites. International Journal of Clothing Science and Technology. https://doi.org/10.1108/09556220910933808

[22] Su, J., Zhang, J. (2016). Preparation and properties of Barium titanate $\left(\mathrm{BaTiO}_{3}\right)$ reinforced high density polyethylene (HDPE) composites for electronic application. Journal of Materials Science: Materials in Electronics, 27(5): 4344-4350. https://doi.org/10.1007/s10854-016-4302-2

[23] Abdalla, N.S., Youssef, M.A., Algarni, H., Awwad, N.S., Kamel, A.H. (2019). All-solid-state poly (vinyl chloride) membrane potentiometric sensor integrated with nanobeads imprinted polymers for sensitive and rapid detection of bispyribac herbicide as organic pollutant. Molecules, 24(4):

712. https://doi.org/10.3390/molecules24040712

[24] Allan, J.T., Easton, E.B. (2016). Polyvinyl chloride composite membranes made with nafion and polysiloxanes for use in electrochemical breath alcohol sensors. Journal of The Electrochemical Society, 163(13): B644. https://doi.org/10.1149/2.0791613jes

[25] Kwak, B., Bae, J. (2020). Integrated design and fabrication of a conductive PDMS sensor and polypyrrole actuator composite. IEEE Robotics and Automation Letters, 5(3): 3753-3760. https://doi.org/10.1109/LRA.2020.2982075

[26] He, Y.L., Zhang, H.J., Zhang, D.X. (2005). Theoretical and experimental study of photo-thermal expansion using an atomic force microscope. Journal of Micromechanics and Microengineering, 15(9): 1637. https://doi.org/10.1088/0960-1317/15/9/004

[27] Zhang, D., Zhang, H., Liu, C., Jiang, J. (2008). Theoretical and experimental study of optothermal expansion and optothermal microactuator. Optics Express, 16(17): 13476-13485. https://doi.org/10.1364/OE.16.013476 